\title{
Modelling, estimation and optimal control issues in cerebrospinal fluid dynamics Kalyan Raman
}

Address: Northwestern University, Medill School, IMC Department, Evanston, IL 60208, USA

Email: Kalyan Raman - k-raman@northwestern.edu

from 53rd Annual Meeting of the Society for Research into Hydrocephalus and Spina Bifida Belfast, UK. 24-27 June 2009

Published: 27 November 2009

Cerebrospinal Fluid Research 2009, 6(Suppl 2):S23 doi:I0.1 I86/1743-8454-6-S2-S23

This abstract is available from: http://www.cerebrospinalfluidresearch.com/content/6/S2/S23

(c) 2009 Raman; licensee BioMed Central Ltd.

\section{Background}

Mathematical models have proved useful in studying the pressure-volume compensation of patients suffering from hydrocephalus, a condition caused by excessive accumulation of cerebrospinal fluid (CSF) in the brain. A standard approach to managing hydrocephalus is through implantation of a shunt. Two important issues in mathematical research on hydrocephalus remain unaddressed-the effect of noise on the nonlinear CSF dynamics and the optimal shunt design for managing hydrocephalus.

\section{Materials and methods}

Natural physical analogies between CSF compensation and an electrical circuit lead to a nonlinear differential equation describing the nature of pressure-volume compensation in CSF dynamics. Physiological measurements of intracranial pressure (ICP) using a constant rate infusion test shows deviations from the deterministic differential equation due to fluctuations caused by noise. These fluctuations raise two fundamental research problems-modelling the effect of noise on CSF dynamics and estimating the resulting model. Furthermore, to improve shunt design, we need an optimal controller that takes the nonlinear dynamics of CSF dynamics into account.

\section{Results}

Visual examination of the noise pattern in the data shows that a Markov process driven by Brownian motion can capture the effects of fluctuations. Such a Markov process finds its mathematical representation in a nonlinear stochastic differential equation (SDE). We solve this nonlin- ear SDE and estimate its parameters through a Kalman filter. We use techniques of modern nonlinear control theory to derive a controller that will keep the patient's ICP at an ideal target level. Theoretically, such a controller can be used to significantly improve existing shunt design.

\section{Conclusion}

We offer the following methodology to advance the state of the art in hydrocephalus research--a nonlinear stochastic differential equation to facilitate theoretical analysis of shunting in hydrocephalus by capturing CSF dynamics more realistically than before, an estimation method based on a sophisticated Kalman filter and an optimal nonlinear controller to stabilize and maintain the patient's ICP at any desired target level.

\section{Acknowledgements}

The author thanks Dr. Marek Czosnyka, Neuroscience Unit, Department of Clinical Neurosciences, University of Cambridge, UK, for supplying the patient data from infusion studies conducted at Addenbrooke's Hospital, Cambridge, UK and for Dr. Czosnyka's insightful comments on this research. The author is grateful to Lisa L. Raman, former National Medical Advisor and Rosemary Batchelor, Senior Advisor Health and Policy Issues, Association for Spina Bifida and Hydrocephalus, ASBAH House, 42 Park Road, Peterborough PEI 2UQ, UK for suggesting the research area and introducing him to researchers studying Hydrocephalus through mathematical modelling. 